\title{
Star Formation History of Dwarfs in Nearby Galaxy Groups
}

L. Makarova ${ }^{1}$, D. Makarov ${ }^{1}$, I. Karachentsev ${ }^{1}$, A. Dolphin ${ }^{2}$, B. Tully ${ }^{3}$, S. Sakai ${ }^{4}$, E. Shaya ${ }^{5}$, L. Rizzi ${ }^{3}$, M. Sharina ${ }^{1}$ and V. Karachentseva ${ }^{6}$

${ }^{1}$ Special Astrophysical Observatory, Russia; ${ }^{2}$ Steward Observatory, USA; ${ }^{3}$ IfA, Univ. of Hawaii, USA; ${ }^{4}$ Univ. Of California, Los Angeles, USA; ${ }^{5}$ Univ of Maryland, USA;

${ }^{6}$ Astronomical Observatory of Kiev, Ukraine

1. The data. We study detailed star formation histories (SFH) of 14 dwarf galaxies in the central parts $(\leqslant 300 \mathrm{Kpc})$ of the two nearby galaxy groups: M $81(\mathrm{D}=3.6 \mathrm{Mpc})$ and Cen $\mathrm{A}(D=3.8 \mathrm{Mpc})$. The images of 7 galaxies were selected from the sample of about 50 nearby dwarf galaxies observed with the Advanced Camera for Surveys (ACS) at the Hubble Space Telescope within our prog. 9771 \& 10235, (PI I.Karachentsev). The rest of the images (ACS and WFPC2) were taken from the Hubble Archive (prog. 9884, 5898 and 6964).

2. The methods and photometry. Photometry of the resolved stars in the galaxies was made with the DOLPHOT package (Dolphin 2002, MNRAS, 332, 91) for crowded field photometry. Photometric distances for all galaxies in the sample were obtained using tip of the red giant branch (TRGB) distance indicator (Lee et al. 1993, ApJ, 417, 553; Sakai et al. 1996, ApJ, 461, 713). We have developed the StarProbe package for quantitative star formation history determination (Makarov \& Makarova 2004, Ap, 47, 229). We create a library of synthetic color-magnitude diagrams from theoretical stellar isochrones, taking the initial mass function, distance to the galaxy, internal and external absorption, and photometric errors into account. The resulting synthetic diagrams are combined linearly and compared quantitatively with photometric data for stars in a galaxy in order to determine star formation rates as a function of age and metallicity.
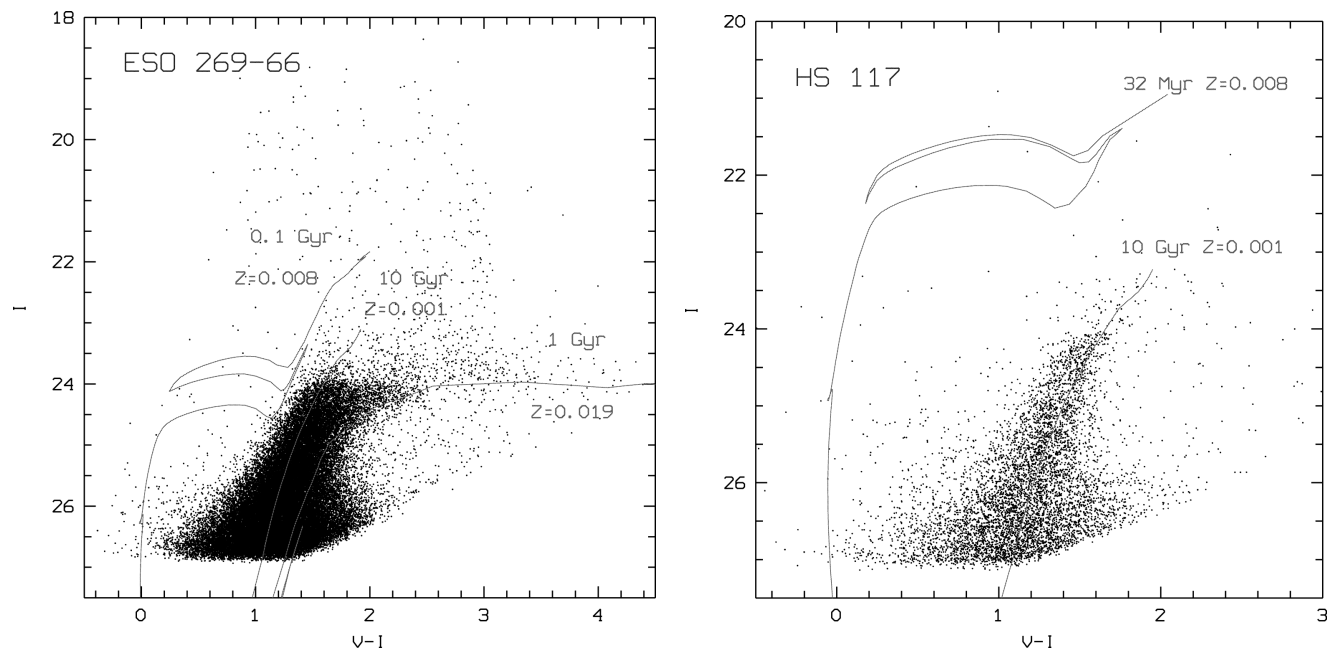

Figure 1. Color-magnitude diagrams of ESO 269-066 ( $D=3.82 \mathrm{Mpc})$ and HS 117 $(D=3.96 \mathrm{Mpc})$ are shown. The galaxies were observed in F606W (1200s) and F814 (900s) filters. The isochrones from Girardi et al. (2000, A\&AS 141, 371) are overplotted. 

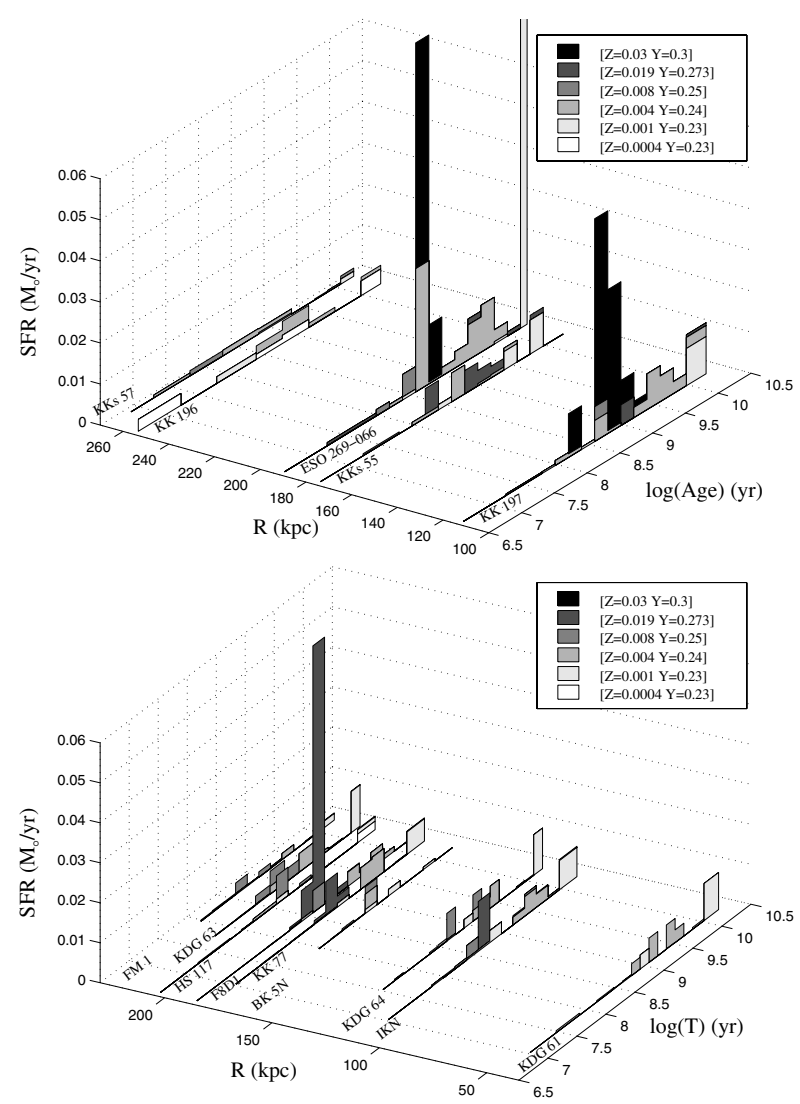

Figure 2. The calculated SFH. The vertical axis shows the star formation rates. Two horizontal axes are the age of star formation episodes and the spatial distance from the main body of the group. Different colors reflect the initial chemical composition of the respective model stars. All the studied galaxies demonstrate several episodes of active star formation.

3. Stellar populations and star formation histories. The color-magnitude diagrams of ESO 269-66 and HS 117 are given in the Fig.1 to represent our sample. The galaxies are populated with mostly old RGB stars. We should also suppose a presence of intermediate age AGB stars. Cen A galaxy ESO 269-66 has unusual RGB color scatter, which reflect active star formation episode with very high metallicity level. Similar features are shown by dSph galaxy KK 197, and, less prominent, KKs 55. Note the similarity of ages $(\sim 300 \mathrm{Myr}-1 \mathrm{Gyr})$ of last star formation episode for most studied galaxies in the M 81 group. The mentioned features of star formation in the central parts of both groups can probably be explained by past strong interactions in the group. 